\title{
Detection of internal tandem duplications in the $F L T 3$ gene by different electrophoretic methods
}

\author{
Tamás Bubán ${ }^{1}$, Katalin Koczok ${ }^{2}$, Róza Földesi², \\ Gabriella Szabó ${ }^{2}$, Andrea Sümegi², Miklós Tanyi ${ }^{3}$, \\ László Szerafin ${ }^{4}$, Miklós Udvardy ${ }^{5}$, János Kappelmayer ${ }^{2}$ \\ and Péter Antal-Szalmás ${ }^{2} * *$
${ }^{1}$ Institute of Internal Medicine, 1st Department of Internal Medicine, University of Debrecen, Medical and Health
Science Center, Debrecen, Hungary
${ }^{2}$ Department of Clinical Biochemistry and Molecular
Pathology, University of Debrecen, Medical and Health
Science Center, Debrecen, Hungary
${ }^{3}$ Department of Surgery, University of Debrecen, Medical
and Health Science Center, Debrecen, Hungary
${ }^{4}$ Department of Hematology, András Jósa County
Hospital, Nyíregyháza, Hungary
${ }^{5}$ Institute of Internal Medicine, 2nd Department of Internal
Medicine, University of Debrecen, Medical and Health
Science Center, Debrecen, Hungary

\begin{abstract}
Background: In acute myeloid leukemia (AML), the internal tandem duplication (ITD) in the juxtamembrane domain of the FLT3 (Fms-like tyrosine kinase 3) gene is one of the most frequent genetic alterations associated with poor prognosis.

Methods: A complex evaluation of the analytical properties of the three most frequently used detection methods - PCR followed by agarose (AGE), polyacrylamide (PAGE) or capillary electrophoresis (CE) - was performed on 95 DNA samples obtained from 73 AML patients.

Results: All the three methods verified the presence of a mutant allele in 20 samples from 18 patients. AGE and PAGE could detect the presence of $1 \%-2 \%$ mutant allele, while the detection limit of CE was $0.28 \%$. However, acceptable reproducibility (inter-assay $\mathrm{CV}<25 \%$ ) of the mutant allele rate determination was only achievable above $1.5 \%$ mutant/ total allele rate. The reproducibility of the ITD size determination by CE was much better, but the ITD size calculated by PeakScanner or GeneScan analysis was 7\% lower as compared to values obtained by DNA sequencing. The presence of multiple ITD was over-estimated by PAGE and AGE due to the formation of heteroduplexes.
\end{abstract}

\footnotetext{
*Corresponding author: Dr. Péter Antal-Szalmás, MD, PhD, Department of Clinical Biochemistry and Molecular Pathology, University of Debrecen, Medical and Health Science Center, Nagyerdei str. 98., 4032 Debrecen, Hungary Phone: +36-52-340006, Fax: +36-52-417631, E-mail: alumni@med.unideb.hu Received May 15, 2011; accepted October 5, 2011; previously published online November 4, 2011
}

Conclusions: This study suggests the use of PCR+CE in the diagnostics and the follow-up of AML patients. The data further supports the importance of proper analytical evaluation of home-made molecular biological diagnostic tests.

Keywords: acute myeloid leukemia; agarose gel electrophoresis; capillary electrophoresis; fms-like tyrosine kinase 3 (FLT3); internal tandem duplication (ITD); polyacrylamide gel electrophoresis.

\section{Introduction}

The Fms-like tyrosine kinase 3 (FLT3) plays an important role in the activation of hematopoetic stem cells. Binding to its ligand results in the induction of different signaling pathways known to regulate cell proliferation, differentiation and apoptosis of these cells. As a result, alteration in functionality of FLT3 can be important in the development of hematological malignancies (1-4). Three different types of activating mutations have been described in the FLT3 gene: a point mutation at nucleotide position 869 of the tyrosine kinase domain (TKD), point mutations in the exons coding the juxtamembrane domain (JM) of the protein and the internal tandem duplication (ITD) in the JM domain (1-4). These mutations are one of the most frequent genetic alterations in acute myeloid leukemia (AML) and show poor prognosis, especially in patients without cytogenetic alterations, those classified as the intermediate prognostic group (3-6).

From the three types of the FLT3 mutations, the FLT3ITD shows the highest frequency and clinically seems to be the most important. Here, a 3-400-base-long DNA fragment is duplicated (rarely triplicated) in the region of exon 14, intron 14 and exon 15 and sometimes short nucleotide additions are also combined with the duplication. In spite of the large nucleotide insertions the reading always remains in frame, resulting in an FLT3 protein with a longer JM domain showing a reduced inhibitory effect on the TKD domain $(1,3-7)$.

The detection of the FLT3-ITDs is based on a PCR using primers designed for the $5^{\prime}$ end of exon 14 and the $3^{\prime}$ end of exon 15. The presence of a longer PCR product compared to that amplified from the wild-type allele shows the presence of the ITD (8). Different electrophoretic methods are available for the analysis of these PCR products. Single stranded conformational polymorphism (9), conformational sensitive gel electrophoresis (10), temperature gradient capillary electrophoresis (11), high-resolution melting analysis (11) and denaturating HPLC (dHPLC) $(12,13)$ are sensitive methods for the detection of small genetic alterations, like ITD, but are rather 
time-consuming and labor-intensive. A simple agarose gel electrophoresis (AGE) is quite economical and easy to handle (7, 8, 14-16). Polyacrylamide gel electrophoresis (PAGE) has higher resolution and sensitivity but is more demanding and, perhaps, is less suitable for routine diagnostic purposes (17-19). Data are somewhat contradictory, but several lines of evidence suggest that the size of the ITD and the rate of mutant allele might have important prognostic information in $\operatorname{AML}(2,13,15,16,20-29)$. Semi-quantitative AGE or PAGE $(14,20)$, dHPLC $(12,13)$, the Agilent Bioanalyser $(30,31)$, capillary electrophoresis (CE; e.g., GeneScan analysis) (13, $17,19,22-29,32,33)$ or real-time quantitative PCR $(34,35)$ can provide information about these parameters.

The application of home-made molecular genetic tests in the field of hemato-oncology is rather wide-spread in routine diagnostic laboratories. The proper evaluation of the analytical properties of these methods prior to their routine application - especially in the case of the quantitative tests - is essential. As such, we compared the analytical properties of PCR combined with AGE, PAGE and CE - the three most frequently used analytical methods - in the detection of FLT3ITD in samples of AML patients.

\section{Materials and methods}

\section{AML patients}

Altogether 73 adult patients (age: $51.7 \pm 18.5$ years, 36 males, 37 females) with AML were enrolled in this study. Patients were followed-up by the 2nd Department of Internal Medicine of the Medical and Health Science Center, University of Debrecen, Debrecen and by the Department of Hematology of the András Jósa County Hospital, Nyíregyháza. Detailed clinical data was available in the case of 51 patients (Supplementary Table 1). The work was assigned by the Local Ethical Committee of the University of Debrecen and informed consent was obtained from each donor. We have complied with the World Medical Association Declaration of Helsinki regarding ethical conduct of research involving human subjects.

\section{Amplification of exon 14, intron 14 and exon 15 of the FLT3 gene}

Genomic DNA was isolated from peripheral blood of AML patients using the QIAmp DNA Blood Minikit (Qiagen, Valencia, CA, USA). The region of exon 14, intron 14 and exon 15 of the FLT3 gene was amplified by a PCR using forward (5'-ATT TAG GTA TGA AAG CCA GC- $\left.3^{\prime}\right)$ and reverse (5'-CTT TCA GCA TTT TGA CGG CAA CC-3') primers (Applied Biosystems, Foster City, CA, USA) (8). For $\mathrm{CE}$ analysis the forward primer was labeled at the 5 -end by 6-carboxy-fluorescein (FAM). Each $25 \mu \mathrm{L}$ reaction included 200-500 ng of genomic DNA, 5 pmol of each primer, $400 \mu \mathrm{M}$ dNTP, $2 \mathrm{mM}$ $\mathrm{MgCl}_{2}$ and $1 \mathrm{U}$ AmpliTaq Gold polymerase (Applied Biosystems). When samples were prepared for the CE analysis, 0.5 pmol of each primers (FAM-labeled forward and non-labeled reverse) and $10 \mathrm{mM}$ $\mathrm{MgCl}_{2}$ was used in the PCR amplification. The PCR was performed using the GeneAmp 2700 PCR system (Applied Biosystems) under the following conditions: denaturation at $95^{\circ} \mathrm{C}$ for $12 \mathrm{~min}$ followed by 35 cycles of denaturation at $94^{\circ} \mathrm{C}$ for $30 \mathrm{~s}$, annealing at $56^{\circ} \mathrm{C}$ for $1 \mathrm{~min}$ and extension at $72^{\circ} \mathrm{C}$ for $2 \mathrm{~min}$. A final extension at $72^{\circ} \mathrm{C}$ for 10 min terminated the amplification.

\section{Agarose gel electrophoresis}

The PCR products $(10 \mu \mathrm{L})$ were loaded with $0.5 \mu \mathrm{L}$ loading dye (bromophenolblue: $4.2 \mathrm{mg} / \mathrm{mL}$; xylen cyanide: $4.2 \mathrm{mg} / \mathrm{mL}$; glycerol: $50 \mathrm{v} / \mathrm{v} \%$; Sigma, St. Louis, MO, USA) into slots of a $10 \times 15 \mathrm{~cm}$ $3 \%$ agarose (Cambrex, East Rutherford, NJ, USA) gel in TBE buffer using the wide-cell horizontal electrophoresis system (Biorad, Hercules, CA, USA). The electrophoresis was performed using a power of $10-11 \mathrm{~V} / \mathrm{cm}$ for $1-2 \mathrm{~h}$. The TBE buffer was changed one to two times during runs in order to prevent excessive warming. The bands were visualized by $0.4 \mu \mathrm{g} / \mathrm{mL}$ ethidium bromide (Sigma) that was mixed into the gel and running buffer.

\section{Polyacrylamide gel electrophoresis}

The PCR products $(10 \mu \mathrm{L})$ were loaded with $0.5 \mu \mathrm{L}$ loading dye into slots of a $15 \times 20 \mathrm{~cm} 6 \%$ polyacrylamide/bisacrylamide (29:1) gel (Biorad) in TAE buffer using the Amersham SE-600 system (Amersham Pharmacia, Piscataway, NJ, USA). The electrophoresis was performed using a power of $10-11 \mathrm{~V} / \mathrm{cm}$ for $1-2 \mathrm{~h}$. The gel was stained after the run by $1 \mu \mathrm{g} / \mathrm{mL}$ ethidium bromide for $20 \mathrm{~min}$ at room temperature.

\section{Isolation of PCR products from the polyacrylamide gel}

The FLT3-ITD PCR products amplified from the wild and mutant alleles were isolated from the polyacrylamide gel by the "crush and soak" procedure. In short, a disposable surgical knife was used to cut out the bands from the gel that were crushed against the wall of a microfuge tube $(1.5 \mathrm{~mL})$ with a disposable pipette tip. To the gel slice we added one to two times of the gel slice volume of elution buffer $\left(\mathrm{NH}_{4}\right.$-acetate $0.5 \mathrm{M} ; \mathrm{MgSO}_{4} 15 \mathrm{mM}$; EDTA $1 \mathrm{mM}, 0.1 \%$ SDS; Sigma). The tube was incubated for $12-16 \mathrm{~h}$ at $37^{\circ} \mathrm{C}$ on a rotary platform and was centrifuged $\left(13,800 \mathrm{~g}, 1 \mathrm{~min},+4^{\circ} \mathrm{C}\right)$. The supernatant was transferred to a new tube while the polyacrylamide pellets were washed again with $0.5 \mathrm{~mL}$ elution buffer. After a repeated centrifugation the second supernatant was combined with the first eluate. The PCR product extracted from the gel was further purified using absolute ethanol and $3 \mathrm{M} \mathrm{Na-acetate} \mathrm{(Sigma).}$

\section{Capillary electrophoresis}

The PCR products containing the 6-FAM-labeled forward primer were diluted 8-times in molecular biology grade water (Promega, Madison, WI, USA). The diluted sample $(1 \mu \mathrm{L})$ was mixed with 10 $\mu \mathrm{L}$ formamide ( $\mathrm{Hi}_{-} \mathrm{Di}^{\mathrm{TM}}$ formamide, Applied Biosystems) and 0.5 $\mu \mathrm{L}$ GeneScan 500-ROX internal size standard (Applied Biosystems) and denaturated for $3 \mathrm{~min}$ at $96^{\circ} \mathrm{C}$. The capillary electrophoresis of the samples was performed using ABI PRISM 310 Genetic Analyzer (Applied Biosystems). The technical parameters were the following: capillary length and diameter: $47 \mathrm{~cm}$ and $50 \mu \mathrm{m}$; capillary temperature: $60^{\circ} \mathrm{C}$; polymer: POP6; run power and time: $15.0 \mathrm{kV}$ and 60 min; injection power and time: $2 \mathrm{kV}$ and $5-15 \mathrm{~s}$. The data collection was performed using the $\mathrm{ABI}$ Prism ${ }^{\mathrm{TM}}$ Collection software (Applied Biosystems), while the runs were analyzed by the PeakScanner 1.0 and in certain cases by the GeneScan ${ }^{\circledR} 3.1$ software (Applied Biosystems). For size determination the local Southern method was applied, unless the size of a certain peak exceeded the upper range of the size standard, when linear regression was used. In samples of de novo cases we accepted signals with relative fluorescence unit (RFU) $>50$ as peaks and in follow-up samples with known peak-localizations even lower RFU values (above 15) were evaluated. The area under the wild-type and mutant peaks was also determined and the 
rate of mutant allele was calculated in two different ways: (i) mutant/ total allele rate $=\mathrm{AUC}_{\text {mutant peak }} /\left(\mathrm{AUC}_{\text {mutant peaks }}+\mathrm{AUC}_{\text {wild-type peak }}\right) \times 100$; and (ii) mutant/wild-type allele rate $=\mathrm{AUC}_{\text {mutant peak }} / \mathrm{AUC}_{\text {wild-type peak }}$.

\section{Bidirectional sequencing of the ITDs}

The PCR products were cleaned from the unbound dNTP, primers and other assay components by a spin column centrifugation (Amicon ${ }^{\circledR}$ Ultra - $0.5 \mathrm{~mL}, 30 \mathrm{~K}$; Millipore, Carrigtowhill, Ireland). A cyclic termination end sequencing reaction was performed using the BigDye Terminator kit (v. 3.1, Applied Biosystems) according to the manufacturers' instruction. The unbound labeled dNTP was removed by centrifugation using the DyeEx ${ }^{\circledR} 2.0$ Spin kit (Qiagen). The purified product $(1 \mu \mathrm{L})$ was mixed with $10 \mu \mathrm{L}$ formamide (Hi-Di ${ }^{\mathrm{TM}}$ formamide) and denaturated for $3 \mathrm{~min}$ at $96^{\circ} \mathrm{C}$. The capillary electrophoresis of the samples was performed using ABI PRISM 310 Genetic Analyzer. The technical parameters were the following: capillary length and diameter: $47 \mathrm{~cm}$ and $50 \mu \mathrm{m}$; capillary temperature: $50^{\circ} \mathrm{C}$; polymer: POP6; run power and time: $15.0 \mathrm{kV}$ and $36 \mathrm{~min}$; injection power and time: $2 \mathrm{kV}$ and $5-15 \mathrm{~s}$. The data collection and analysis was performed using the $\mathrm{ABI}$ Prism ${ }^{\mathrm{TM}}$ Collection and Sequencing Analysis 3.4.1 softwares.

\section{Identification of FLT3-TKD mutations}

The determination of the presence of the FLT3-TKD mutations - as part of the general diagnostic procedure for AML patients - was performed by a separate PCR+RFLP technique based on the article of Yamamoto et al. (36). The frequency of FLT3-TKD mutation was 2/75 AML patients; combined FLT3-ITD and -TKD mutations were not present. In order to make the study population more homogenous we have enrolled in the analysis only the 73 individuals without FLT3-TKD mutations.

\section{Statistical analysis}

The descriptive statistics were calculated with the help of Microsoft Excel software. The differences in the laboratory parameters between the ITD-positive and -negative AML patients were evaluated by the Mann-Whitney U-test, the gender distribution by the $\chi^{2}$-test, while the survival data between these groups was compared by the Kaplan-Meier analysis. The correlation between the different ways of ITD size determination was evaluated by Pearson's regression analysis. Significant difference was considered in the case of $\mathrm{p}<0.05$. Statistical analysis was performed with the help of the "Statistica" software.

\section{Results}

\section{Comparison of the performance of $A G E$, PAGE and CE on FLT3-ITD detection in samples of patients with AML}

Altogether 95 DNA samples of 73 adult patients with AML were tested for the presence of FLT3-ITD using the three different electrophoretic methods. Two independent samples were tested in the case of 18 and three samples in the case of two patients. We could detect the presence of at least one mutant allele in 20 samples of 18 patients, irrespective of the detection method used. Detailed clinical data was available only in the case of 51 patients and the ITD-positivity rate was 12/51 in this cohort. Similarly to previously published data (1-6) the FLT3-ITD positive patients had higher white blood cell count and LDH values and the overall and disease-free survival period was shorter in this group (Supplementary Table 1).

Based on the CE method the size of the ITD detected in the positive samples varied between 9 and 178 bp (median $40 \mathrm{bp}$ ), while the rate of the mutant to wild type or the mutant to total alleles was 0.006-3.714 (median: 0.144) and $0.48 \%-77.8 \%$ (median: $12.5 \%$ ), respectively. In nine of the 18 ITD-positive patients more than one type of the mutant allele could be detected (multiple ITDs). The number of the cases with two and three mutant peaks on $\mathrm{CE}$ was seven and two, respectively.

Although all three detection methods could identify the FLT3-ITD positive samples, there was a discrepancy concerning the number, size and intensity of the mutant alleles present (Figure 1). Consequently, the analytical properties of the three electrophoretic methods used for FLT3-ITD detection were evaluated.

\section{Evaluation of the analytical properties of AGE, PAGE and CE on FLT3-ITD detection: reproducibility}

In order to evaluate the intra- and inter-assay reproducibility of the tested electrophoretic methods two FLT3-ITD positive samples (P35 and P80) were amplified 10-times in the same PCR assay on the same day and once daily on the following 9 days. With CE, sample P35 contained one major mutant peak and a hardly visible minor peak at $387 \mathrm{bp}$ and $339 \mathrm{bp}$, respectively. In sample P80, we detected a major mutant peak at $355 \mathrm{bp}$ and a very faint peak at 398 bp (Supplementary Figure 1).

Table 1 contains the reproducibility data of the size quantitation by CE. Here, the intra-assay variability was calculated in two ways. First, the two positive samples were amplified 10-times in the same PCR on the same day and then each PCR product was injected independently into the capillary of the ABI310 analyzer (Table 1, "10 cups"). Second, one of the 10 PCR products from both samples was injected 10-times subsequently into the analyzer (Table 1 , "1 cup"). Regarding the size of the individual wild-type and mutant peaks, we observed very good intra- and inter-assay reproducibility (the CV\% was typically below $0.2 \%$ ). The size of the ITD was calculated as the size of the mutant allele minus the size of the wild-type allele. The intra- and inter-assay CV\% of ITD was below $1 \%$ and $2 \%$, respectively, if the ITD size was larger (above 24 bp; sample P35-ITD2; sample P80-ITD1 and -ITD2). In the case of ITD1 of sample P35 a smaller, 9 bp insertion could be observed with a relatively higher intra- and inter-assay CV\% (7\% and $11 \%)$.

Concerning the quantitation of the mutant/wild-type and mutant/total allele rate we obtained variable results (Table $2)$. In the case of sample P35-ITD2 and sample P80-ITD1 the mutant/total allele rate was $65 \%$ and $12 \%$, and the intraand inter-assay $\mathrm{CV} \%$ s were below $5 \%$. The reproducibility was worse when the mutant/total allele rate was $\sim 1.2 \%$ $1.5 \%$ (sample P80-ITD2), showing an intra-assay CV\% of $10 \%-14 \%$ and an inter-assay CV\% of $25 \%$. When the mutant/ 

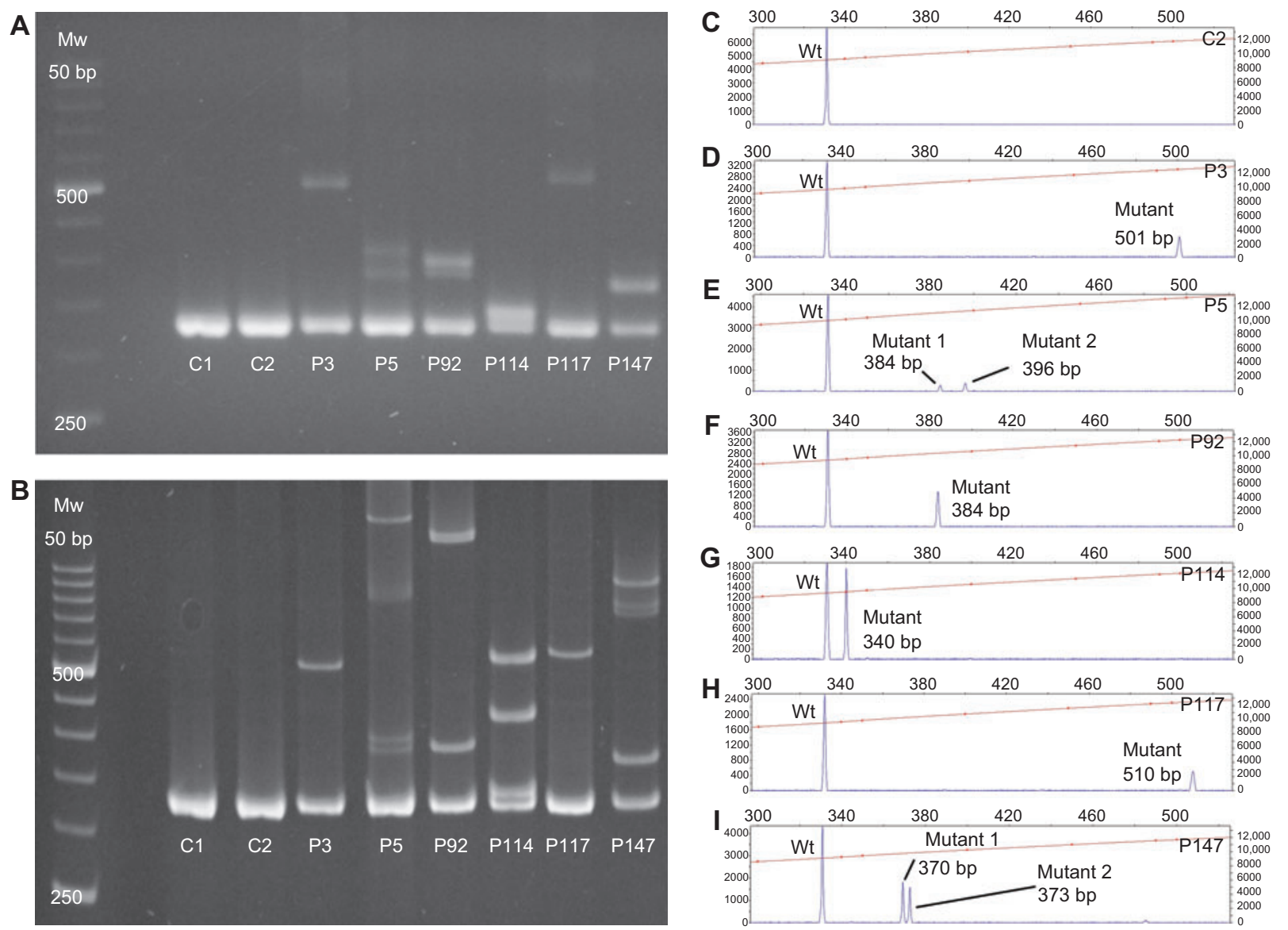

Figure 1 Analysis of FLT3-ITD-positive and -negative DNA samples by three different electrophoretic methods.

Genomic DNA was amplified as described in Materials and methods. The PCR products (10 $\mu \mathrm{L})$ were loaded on 3\% agarose (A) and 6\% polyacrylamide gel (B). For capillary electrophoretic analysis a different PCR protocol was used with a fluorescently labeled forward primer. The samples were measured by an ABI310 analyzer, the size of the PCR products were determined by the PeakScanner software based on a ROX-500 size-standard (C, D, E, F, G, H, I). The size of the PCR product amplified from the FLT3 wide-type allele is 331 bp, and any fragments larger than this indicate the presence of ITD. (C, controls; P, patients.)

total rate was below $1 \%$ (sample P35-ITD1) the reproducibility was poor: $\mathrm{CV} \%$ s were above $50 \%$.

Although quantitative data was not gathered from the agarose and polyacrylamide gel electrophoretograms in our study, we performed the reproducibility analysis in the case of these methods as well. Evaluating with agarose and polyacrylamide gel electrophoresis, the major mutant bands were clearly visible with similar size and intensity for each day and each run while the minor mutant bands were absent (data not shown).

\section{Evaluation of the analytical properties of AGE, PAGE and CE on FLT3-ITD detection: analytical sensitivity}

As a next step the detection limit of the three different electrophoretic methods was compared. The DNA samples of ITD-positive patients (P35 or P80) were serially diluted in an ITD-negative DNA. The DNA concentration in the FLT3positive and negative samples was equal. The rate of the mutant to total alleles in the original samples was determined by $\mathrm{CE}$ and PeakScanner analysis and this rate in the diluted
DNA samples was calculated based on the initial value and the dilution factor (Figure 2A and B). These DNA mixes were amplified using unlabeled primers in the FLT3-PCR and 10 $\mu \mathrm{L}$ of the PCR products were loaded into an agarose or a polyacrylamide gel (Figure 2A and B). The presence of the dominant mutant allele (mutant 2 in the case of P35) could be detected typically at $1 \%-2 \%$ both on agarose and polyacrylamide gel (Figure 2A and B). The minor mutant alleles were not detectable by these methods.

The serially diluted ITD-positive DNA was also tested using a fluorescently labeled forward and unlabeled reverse primer during PCR, followed by denaturation of the PCR products and analysis by $\mathrm{CE}$. The measured rate of the mutant to wild-type (Figure 2D) or mutant to total (Figure 2C) alleles was determined by PeakScanner analysis. These rates were expressed as a function of the calculated mutant rates in the diluted samples. As two different samples in different dilutions were tested, the data of three independent experiments was pooled (Figure 2C and D). In order to find the lowest point of the linear part of the dilution curves a running cut- 
Table 1 Analytical performance of FLT3-ITD size quantitation done by capillary electrophoresis.

\begin{tabular}{|c|c|c|c|c|c|}
\hline & Wild-type allele ${ }^{a, b}$ & Mutant allele $1^{\mathrm{a}, \mathrm{b}}$ & Mutant allele $2^{\mathrm{a}, \mathrm{b}}$ & ITD $1^{\mathrm{b}}$ & ITD $2^{\text {b }}$ \\
\hline \multicolumn{6}{|l|}{ Sample P35 } \\
\hline Intra-assay variation $(1 \text { cup })^{\mathrm{c}}$ & $330.9 \pm 0.2(0.1 \%)^{\mathrm{d}}$ & $339.2 \pm 0.6(0.2 \%)$ & $387.2 \pm 0.1(0.1 \%)$ & $8.3 \pm 0.6(7.2 \%)$ & $56.1 \pm 0.3(0.5 \%)$ \\
\hline Intra-assay variation $(10 \text { cups })^{c}$ & $331.2 \pm 0.1(0.1 \%)$ & $338.9 \pm 0.4(0.1 \%)$ & $386.9 \pm 0.1(0.1 \%)$ & $7.7 \pm 0.5(6.5 \%)$ & $55.7 \pm 0.1(0.2 \%)$ \\
\hline Inter-assay variation $^{\mathrm{e}}$ & $330.6 \pm 0.5(0.2 \%)$ & $339.7 \pm 0.6(0.2 \%)$ & $387.1 \pm 0.1(0.1 \%)$ & $9.1 \pm 1.0(11.0 \%)$ & $56.5 \pm 0.6(1.1 \%)$ \\
\hline \multicolumn{6}{|l|}{ Sample P80a } \\
\hline Intra-assay variation $(1 \mathrm{cup})^{\mathrm{c}}$ & $330.8 \pm 0.2(0.1 \%)^{\mathrm{d}}$ & $355.2 \pm 0.1(0.1 \%)$ & $1 \%)$ & 24.4 & $67.6 \pm 0.3(0.4 \%)$ \\
\hline Intra-assay variation $(10 \text { cups })^{\mathrm{c}}$ & $331.0 \pm 0.2(0.1 \%)$ & $355.2 \pm 0.1(0.1 \%)$ & $398.1 \pm 0.2(0.1 \%)$ & $24.1 \pm 0.2(0.8 \%)$ & $67.1 \pm 0.4(0.6 \%)$ \\
\hline Inter-assay variation $^{\mathrm{d}}$ & $330.3 \pm 0.5(0.2 \%)$ & $355.2 \pm 0.1(0.1 \%)$ & $398.5 \pm 0.3(0.1 \%)$ & $24.8 \pm 0.4(1.6 \%)$ & $68.1 \pm 0.7(1.0 \%)$ \\
\hline
\end{tabular}

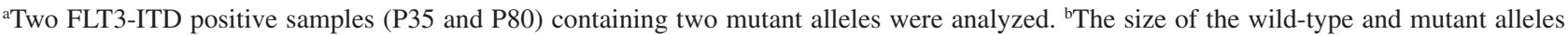
was determined by CE using the ABI310 analyzer and the PeakScanner software. The size of the ITDs was calculated as the size of the mutant alleles minus the size of the wild-type allele. 'The intra-assay variability was calculated in two ways. First, the two positive samples were amplified 10-times in the same PCR on the same day and then each PCR product was injected independently into the capillary of the ABI310 analyzer ("10 cups"). Second, one of the 10 PCR products from both samples was injected 10-times subsequently into the analyzer ("1 cup"). 'Data presented show the mean \pm SD values calculated from the 10 determinations. CV\% (presented in brackets) was calculated as "SD/mean $\times 100$ ". 'The two FLT3-ITD positive samples were amplified and analyzed once on 10 subsequent days.

off from $0.1 \%$ to $0.5 \%$ for the calculated mutant/total allele rate (Figure 2C) and 0.001-0.005 for the calculated mutant/ wild-type allele rate (Figure 2D) was used and a linear regression line was inserted upon the data points above the actual cutoff. The best regression coefficient was observed in the case of $0.2 \%$ and 0.002 cut-offs for the calculated mutant/total and mutant/wild-type allele rates $(\mathrm{R}=0.989$ and $\mathrm{R}=0.990$, respectively). The analytical sensitivity of the assay was calculated using the 10 data points with the lowest calculated mutant allele rates. The mean $+3 \mathrm{SD}$ of these values were $0.28 \%$ and 0.0028 , defining the detection limit of the measured mutant/ total and mutant/wild-type allele rates (Figure 2C and D).

\section{Evaluation of the analytical properties of AGE, PAGE and CE on FLT3-ITD detection: resolution}

A 9 bp difference between the PCR products amplified from the wild-type and mutant alleles in sample P114 could be detected by all of the applied electrophoretic methods (Figure 1A, B and G). On the contrary, in the case of sample P147, a 370 and a 373 bp mutant allele could be detected by CE, but this 3 bp difference could not be resolved by agarose and polyacrylamide gel electrophoresis (Figure 1A, B and I).

\section{Analysis of multiple bands detected in FLT3-ITD positive samples by PAGE and AGE}

In the 20 FLT3-ITD positive DNA samples, we could detect two, three and four mutant bands with higher molecular weight in 12, three and two cases and two, three and zero cases using PAGE and AGE, respectively. The majority of these larger "extra" bands were not present on the CE runs (Figure 1).

In order to reveal their nature - in the case of Patient $N^{\circ} 55$ - the wild-type (band 1=331 bp), mutant 1 (band 2=approx. $350 \mathrm{bp}$ ) and mutant 2 (band $3=$ approx. $550 \mathrm{bp}$ ) bands were

Table 2 Analytical performance of FLT3-ITD rate quantitation done by capillary electrophoresis.

\begin{tabular}{|c|c|c|c|c|}
\hline & Mutant $1 /$ total, $\%$ a,b & Mutant $2 /$ total, $\%$ a,b & Mutant 1/wild type ${ }^{\mathrm{a}, \mathrm{b}}$ & Mutant 2/wild type $\mathrm{e}^{\mathrm{a}, \mathrm{b}}$ \\
\hline \multicolumn{5}{|l|}{ Sample P35a } \\
\hline Intra-assay variation $(1 \text { cup })^{\mathrm{c}}$ & $0.39 \pm 0.20(51.3 \%)^{\mathrm{d}}$ & $63.9 \pm 0.5(0.8 \%)$ & $0.011 \pm 0.006(54.5 \%)$ & $1.790 \pm 0.035(2.0 \%)$ \\
\hline Intra-assay variation $(10 \text { cups })^{\mathrm{c}}$ & $0.43 \pm 0.34(79.1 \%)$ & $65.3 \pm 1.6(2.5 \%)$ & $0.012 \pm 0.009(75.0 \%)$ & $1.911 \pm 0.114(6.0 \%)$ \\
\hline Inter-assay variation $^{\mathrm{e}}$ & $0.63 \pm 0.36(57.1 \%)$ & $65.7 \pm 2.4(3.7 \%)$ & $0.020 \pm 0.012(60.0 \%)$ & $2.052 \pm 0.203(9.9 \%)$ \\
\hline \multicolumn{5}{|l|}{ Sample P80a } \\
\hline Intra-assay variation $(1 \text { cup })^{\mathrm{c}}$ & $11.9 \pm 0.1(0.8 \%)^{\mathrm{d}}$ & $1.5 \pm 0.1(6.7 \%)$ & $0.138 \pm 0.001(0.7 \%)$ & $0.018 \pm 0.002(11.1 \%)$ \\
\hline Intra-assay variation $(10 \mathrm{cups})^{\mathrm{c}}$ & $12.6 \pm 0.2(1.6 \%)$ & $1.2 \pm 0.1(8.3 \%)$ & $0.146 \pm 0.003(2.1 \%)$ & $0.014 \pm 0.002(14.3 \%)$ \\
\hline Inter-assay variation $^{\mathrm{e}}$ & $12.5 \pm 0.5(4.0 \%)$ & $1.3 \pm 0.3(23.1 \%)$ & $0.144 \pm 0.007(4.9 \%)$ & $0.016 \pm 0.004(25.0 \%)$ \\
\hline
\end{tabular}

aTwo FLT3-ITD positive samples (P35 and P80) containing two mutant alleles were analyzed. ${ }^{\text {b}}$ The area under the peaks (AUC) of the wildtype and mutant alleles was determined by $\mathrm{CE}$ using the ABI310 analyzer and the PeakScanner software. The rate of the mutant alleles was calculated as $\mathrm{AUC}_{\text {mutant allele }} / \mathrm{AUC}_{\text {mutant+wild-type alleles }}$ or as $\mathrm{AUC}_{\text {mutant allele }} / \mathrm{AUC}_{\text {wild-type allele }}{ }^{\mathrm{c}}$ The intra-assay variability was calculated in two ways. First, the two positive samples were amplified 10-times in the same PCR on the same day and then each PCR product was injected independently into the capillary of the ABI310 analyzer ("10 cups"). Second, one of the 10 PCR products from both samples was injected 10-times subsequently into the analyzer ("1 cup"). 'Data presented show the mean \pm SD values calculated from the 10 determinations. CV\% (presented in brackets) was

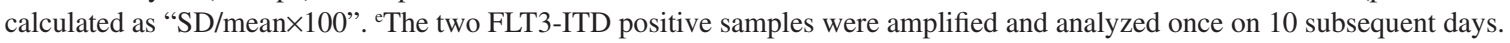




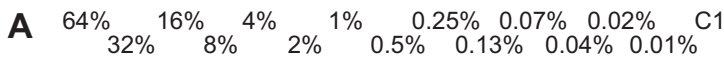
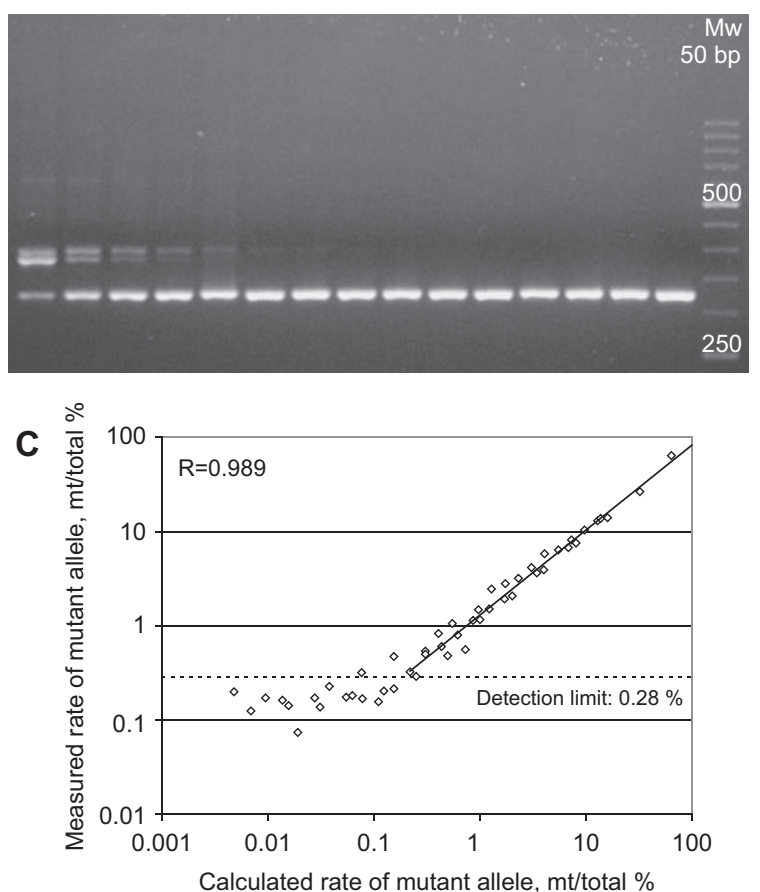

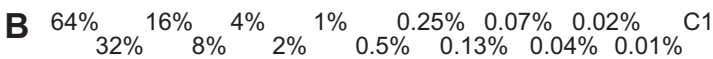

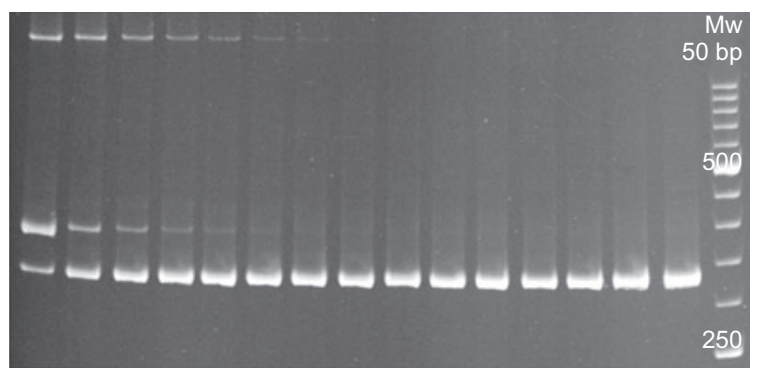

D

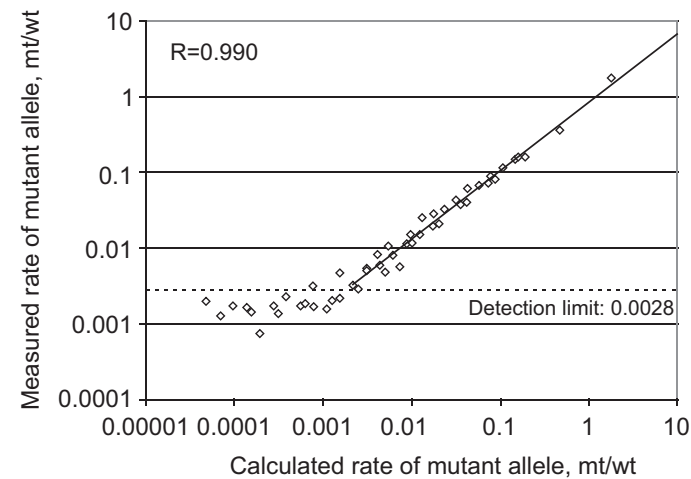

Figure 2 Sensitivity of agarose-, polyacrylamide- and capillary electrophoresis used for identification of FLT3-ITD.

A serial dilution of an ITD-positive DNA sample (P35) was prepared using an ITD-negative DNA with the same concentration. These mixes were amplified as described in Materials and methods and $10 \mu \mathrm{L}$ of the PCR product was loaded into an agarose (3\%, A) and a polyacrylamide $(6 \%, \mathrm{~B})$ gel. The numbers above the lanes show the mutant/total allele rates calculated in the DNA mixes. The size of the PCR products amplified from the FLT3 wild-type and mutant allele are 331 and 387 bp, respectively. For capillary electrophoretic analysis a different PCR was applied with a fluorescently labeled forward primer using the DNA dilution series described above. The area under the wild-type and mutant peaks was determined by the PeakScanner software and the measured AUC rates (C: mutant/total, D: mutant/wild type) were expressed against the calculated rates. The regression lines on part $\mathrm{C}$ and $\mathrm{D}$ show the best fit on the points above $0.2 \%$ mutant/total and 0.002 mutant/wild-type rate, while the detection cut-offs were defined as the mean $+3 \mathrm{SD}$ of the lowest 10 points, respectively. Parts A and B show one representative experiment out of three, while parts $\mathrm{C}$ and $\mathrm{D}$ present the pooled data of three independent determinations.

cut from the polyacrylamide gel (Figure 3A, lane ' $\mathrm{O}$ '). Their DNA content was extracted and was further tested. First the DNA sequence was determined by bidirectional sequencing. Comparison of the wild-type (band 1) and mutant 1 (band 2) sequences revealed a 21-bp-long duplication in intron14/ exon15 (c.1773_1793dup21) in mutant 1 (Figure 3C and D). In mutant 2 (band 3 ) the mixture of the two sequences could be observed even after repeated excision and amplification of this band (Figure 3E).

This can suggest that the mutant band 2 is a heteroduplex of the wild-type and the mutant 1 bands. In order to prove this hypothesis two experiments were performed. First, the DNA of the wild-type and the mutant 1 bands isolated from the gel was mixed and amplified together in the FLT3-ITD PCR (Figure 3A, lane "1/2"). Second, the two isolated DNA were amplified independently by the FLT3-PCR, and then mixed, denaturated at $95^{\circ} \mathrm{C}$ for $10 \mathrm{~min}$ and cooled down slowly $\left(1^{\circ} \mathrm{C} /\right.$ $\min$ ) to $4^{\circ} \mathrm{C}$ (Figure $3 \mathrm{~A}$, lane “ $\mathrm{d} / \mathrm{r}$ "). In both cases the wildtype, mutant 1 and mutant 2 bands were visible by PAGE.

Finally, the presence of the two mutant bands was also analyzed by a separate PCR using fluorescence primers and CE by ABI 310 analyzer. Here, the heteroduplexes were first eliminated by denaturating the PCR products by formamide and heating before loading into the capillary. CE revealed the presence of the wild-type allele (331 bp) and only one peak was amplified from a mutant allele (350 bp; Figure 3B).

\section{Comparison of FLT3-ITD size quantitation determined by CE and DNA sequencing}

It is known from previous publications that the FLT3-ITDs always result in an in-frame genetic alteration, i.e., the ITD size is a multiple of $3(1,3,4)$. Based on our data the ITD size determination performed by $\mathrm{CE}$ revealed values that did not correspond strictly to this rule. Therefore, in 10 cases we directly sequenced the DNA of ITD-positive samples and compared the number of duplicated/inserted nucleotides in the dominant mutant allele to the ITD size determined by CE (Table 3 ). The correlation between the two methods was very strong $(\mathrm{R}=0.999)$, but the size of the duplication/insertion was $7 \%$ lower if it was determined by CE ( Size $_{\text {sequencing }}=1.07 \times$ Size $_{\mathrm{CE}}+0.5$ ) (Supplementary Figure 2A). This alteration was not peculiar to the PeakScanner software, which is a freeware program for PCR fragment analysis, as after analyzing the 

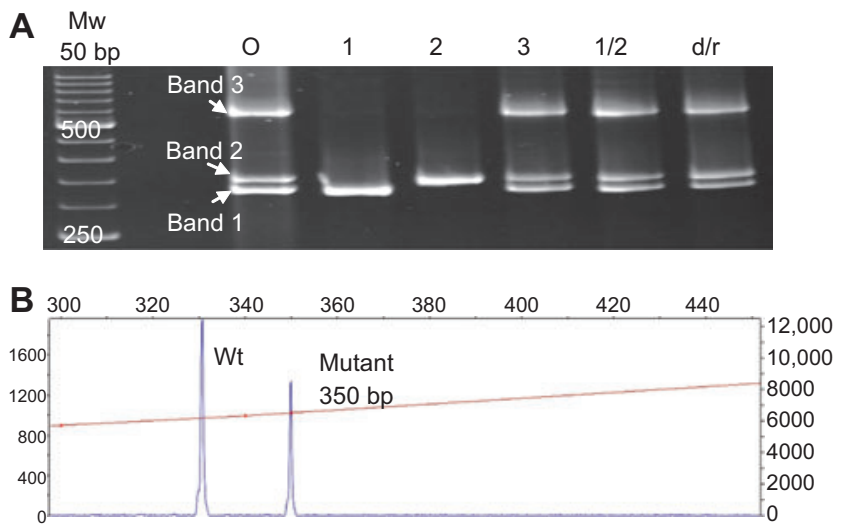

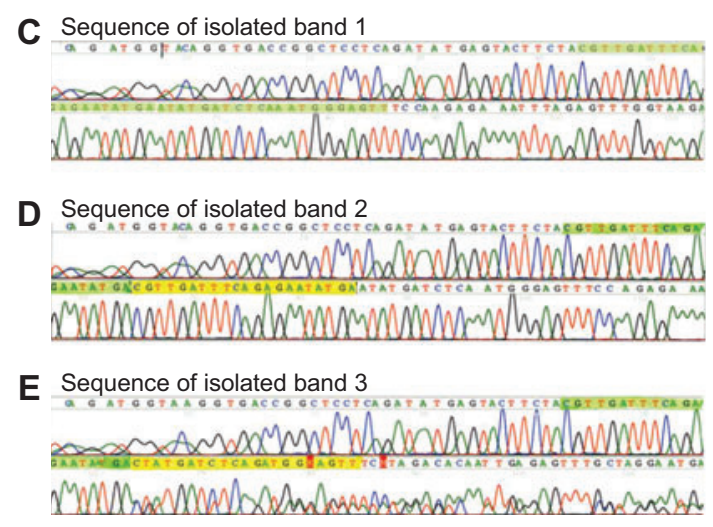

Figure 3 Larger extra bands observed in the presence of ITD on polyacrylamide gel electrophoretograms are heteroduplexes of PCR products amplified from the wild-type and the mutant alleles.

DNA sample of P55 was amplified using the PCR described in Materials and methods. The PCR product of the wild-type allele (331 bp, band 1) and two extra bands with higher molecular weight (band 2 and 3) could be observed using polyacrylamide gel electrophoresis (lane "O", part A). The three bands were excised from the gel and were reamplified separately using the same PCR (Lane " 1 ", " 2 " and " 3 ", part A). Then the isolated bands 1 and 2 were mixed in equal concentrations and were amplified again in the same PCR reaction (lane " $1 / 2$ ", part A) or the isolated bands 1 and 2 were amplified independently, were mixed in equal rate and were denaturated at $+95^{\circ} \mathrm{C}$ followed by slow cooling to $+4^{\circ} \mathrm{C}$ (lane "d/r", part A). The isolated and reamplified bands were also sequenced. Band 1 showed the wild-type sequence (C), band 2 had a 21 bp ITD (c.1773_1793dup21; D), while the sequence of band 3 was the mixture of the two former ones (E). Capillary electrophoresis of fluorescently labeled and denaturated PCR products revealed only the wild-type and one mutant peak of $350 \mathrm{bp}$ (B).

raw data of 27 ITD peaks of 17 positive samples with the GeneScan software perfectly matching results were achieved $\left(\right.$ Size $_{\text {GeneScan }}=1.0 \times$ Size $_{\text {PeakScanner }}+0.1 ; \mathrm{R}=0.999 ;$ Supplementary Figure 2B).

\section{Discussion}

FLT3-ITD is one of the most frequent genetic alterations in adult patients suffering from AML. In our study the rate of ITD-positivity in the whole study cohort was $24.7 \%$ (18/73), showing good agreement with the majority of published data $(3,4,6,7)$. Moreover, the rate of ITD-positive samples was similar for all the three detection methods - AGE, PAGE,
CE - used, indicating a comparable analytical sensitivity for the three techniques. On the other hand, some minor mutant bands detectable by CE were not visible on the agarose or polyacrylamide gels. Here, the detection limit of the assays determined by serial dilution of an ITD-positive DNA sample - was $1 \%-2 \%$ for AGE and PAGE and $0.28 \%$ for CE. In the majority of the former FLT3-ITD publications similar detection sensitivities were found in the case of AGE and PAGE $(17,19)$. Concerning $\mathrm{CE}$ a detection threshold of $0.8 \%-2 \%$ was described for FLT3-ITD quantitation in five publications (18, $37-40)$, while $3 \%-10 \%$ in another four papers $(25,41-43)$. The somewhat lower detection limit of CE observed in our study might be explained by the low RFU threshold used for peak identification by us. In new samples we accepted signals

Table 3 Comparison of FLT3-ITD size determined by PaekScanner and GeneScan analysis and DNA sequencing.

\begin{tabular}{llll}
\hline Sample $^{\mathrm{a}}$ & DNA sequencing & PeakScanner $^{\mathrm{b}}$ & GeneScan $^{\mathrm{b}}$ \\
\hline P13 & c.1773_1793dup21 & $19.2 \pm 0.3 \mathrm{bp}$ & $19.7 \pm 0.4 \mathrm{bp}$ \\
P16 & c.1742_1777dup36 & $32.7 \pm 0.2 \mathrm{bp}$ & $33.4 \pm 0.9 \mathrm{bp}$ \\
P35 & c.1776_1833dup58+c.1833_1834insCC & $56.5 \pm 0.6 \mathrm{bp}$ & $56.9 \pm 0.5 \mathrm{bp}$ \\
P55 & c.1773_1793dup21 & $19.1 \pm 0.2 \mathrm{bp}$ & $18.9 \pm 0.2 \mathrm{bp}$ \\
P58 & c.1772_1833dup62+c.1833_1834insGAGA & $61.5 \pm 0.2 \mathrm{bp}$ & $60.9 \pm 1.4 \mathrm{bp}$ \\
P80 & c.1734G>A+c.1734_1735insCAACCAACCAACCATGCGAGGGCCTCC & $24.8 \pm 0.4 \mathrm{bp}$ & $25.0 \pm 0.7 \mathrm{bp}$ \\
P92 & c.1790_1835dup46+c.1835_1836insCCTCCCCACGG & $52.5 \pm 0.3 \mathrm{bp}$ & $52.5 \pm 0.3 \mathrm{bp}$ \\
P113 & c.1762_1827dup66 & $61.3 \pm 1.1 \mathrm{bp}$ & $61.5 \pm 1.0 \mathrm{bp}$ \\
P114 & c.1788_1789insGGGGTCCCT & $9.0 \pm 0.1 \mathrm{bp}$ & $9.1 \pm 0.1 \mathrm{bp}$ \\
P125 & c.1786_1837+3dup55+c.1837+3_1837+4insCC & $52.3 \pm 0.6 \mathrm{bp}$ & $52.0 \pm 1.3 \mathrm{bp}$ \\
\hline
\end{tabular}

aThe DNA of 10 ITD-positive samples was directly sequenced and the location and number of duplicated/inserted nucleotides in the dominant mutant allele was determined. ${ }^{\mathrm{b}}$ The size of the wild-type and mutant alleles was determined by CE using the ABI310 analyzer and the PeakScanner or GeneScan programs. The size of the ITDs was calculated as the size of the mutant alleles minus the size of the wild-type allele. ${ }^{c}$ Data presented are mean \pm SD of 3-10 independent determinations. 
with RFU $>50$ as peaks and in follow-up samples with known peak-localizations even RFU values above 15 were accepted. In this way - based on a maximal RFU signal of $6000-a$ detection limit below $1 \%$ could be obtained. Only some information about the RFU threshold values is available in former FLT3-ITD studies. Murphy et al. (42) used RFU=150 as a threshold, while Lin et al. (18) mentioned RFU=110 as a very low peak height reaching a maximal FLT3-ITD detection sensitivity of $2 \%-3 \%$. To ensure the presence of ITD-mutants with low signal intensity, analysis was repeated and declared positive only if the result was reproducible.

Although mutant alleles with very low rates were not detectable by AGE and PAGE, several extra bands with larger size were visible that were absent in the electrophoretograms of CE. We performed a detailed analysis of the isolated PCR products amplified from the wild-type and mutant alleles using DNA sequencing and heteroduplex testing, and could prove that the large extra bands corresponded to the heteroduplexes formed by the wild-type and mutant alleles. Previous publications also suggest this phenomenon $(30,42,43)$. Based on the absence of mutant alleles with very low rates and the presence of heteroduplexes on AGE and PAGE, the method that is suitable for the reliable detection of multiplex ITDs is CE.

By examining our patient samples we could compare the resolution of the applied electrophoretic methods, too. While any of the three methods could differentiate between bands/peaks which were 9 bp apart, a 3-bp-difference could be resolved only by CE. A similar superior resolution of CE-based FLT3-ITD analysis was observed in two previous publications $(18,27)$, further supporting the importance of this technique in multiple ITD detection. The presence of multiple ITDs - determined by CE - in our study was $50 \%$ (9/18), showing somewhat higher values compared to the previously published $11 \%-32 \%(7,13,19-22,27,33,42,44)$. This might be explained by the lower threshold used for the definition of "peaks" on the CE electrophoretograms.

Concerning the size of ITD, basically three different techniques have been used for its quantitation so far. DNA sequencing $(7,14,21,23,26,27,29)$, CE $(22,24,33,40,43-$ $45)$ and application of the Agilent Bioanalyzer (30, 31, 46) showed ITD sizes varying from 3 to $400 \mathrm{bp}$, with a median changing between 24 and $70 \mathrm{bp}$. Our results were within these limits, as the observed ITD size range was 9-178 bp, with a median of $40 \mathrm{bp}$. Our data showed a good reproducibility of the ITD size determination by $\mathrm{CE}$, as an inter-assay $\mathrm{CV} \%$ of $11 \%$ was achievable even in the case of the shortest insertion ( $9 \mathrm{bp})$. The number of duplicated/inserted nucleotides was not divisible by three in every case, this has been reported in two former publications $(30,45)$, but not in other studies using Agilent Analyzer or CE. This phenomenon can be explained by the observed variation of about \pm 0.5 bp in the size of the wild-type and mutant peaks on the CE electrophoretograms that can result in a \pm 1.0 bp change in the ITD-size. As such, the method that can provide the exact ITD size is direct sequencing of patient DNA samples or sequencing the isolated - and cloned - ITD fragments. Following the former approach we sequenced 10 samples with ITD and always found the duplication/insertion inframe, but these ITD sizes were slightly larger as compared to data obtained by CE and PeakScanner or GeneScan analysis. Based on literature data and our own experience, we suggest the usage of DNA sequencing for exact ITD size determination and especially because recent data emphasized that even the location of the duplication/insertion might have prognostic importance (13).

Five different methods were used so far to determine the rate of the mutant allele in samples of AML patients: semiquantitative AGE or PAGE $(14,20)$, dHPLC $(12,13)$, Agilent Bioanalyzer $(30,31,46)$, real-time quantitative PCR $(34,35)$ and $\mathrm{CE}(13,17,19,22-29,32,33)$. Concerning the expression of the results two major approaches were described in literature; the mutant/wild-type $(13,23-28,32)$ or the mutant/ total allele rate $(17,19,20,22,30,46)$ was used with similar frequency. As such we calculated both rates in our analysis. The median of the mutant/wild-type allele rate in our patient group was 0.144 (range: $0.006-3.714$ ), while median of the mutant/total allele rate showed $12.5 \%$ (range: $0.48 \%-77.8 \%$ ), this seems to be somewhat lower than previously published data (range for all data and for median of the mutant/wild-type rate: $0.008-32.56$ and $0.530-0.836$; range for all data and for median of the mutant/total rate: $1 \%-100 \%$ and $30 \%-47 \%$ ). Most probably this can be explained again by the lower RFU limit used for peak definition in our study. Concerning the reproducibility of these determinations our results indicated that the mutant/wild-type and the mutant/total allele rate can be determined with an inter-assay $\mathrm{CV} \%<25 \%$ above 0.020 and $1.5 \%$, respectively, giving the quantitation limit of these determinations.

The clinical importance of the presence of multiple ITDs and the ITD-size determination seems to be conflicting, and cannot be suggested for routine diagnostic application at the moment $(13,20-24)$. With regards to the clinical importance of the mutant allele rate quantitation, literature data is more solid and several articles showed that higher ITD-allele rate - and especially the loss of the wild-type allele $(15,16)$ - is associated with worse overall- and disease-free survival and a higher relapse rate in $\operatorname{AML}(3,13,20,22,24-29)$. Here, the major problem is how to define "high" rate. Mostly the median of the allele rate values measured in a patient group was used as cut-off, but this varied from 0.530 to 0.836 for the mutant/wild-type allele rate and 30\%-47\% in the case of the mutant/total rate. Based on literature data and our own experience, we suggest the usage of the mutant/total allele rate that gives more clear information to the clinician as it can be scaled from $0 \%$ to $100 \%$. The development of a general diagnostic cut-off needs further effort, either by pooling the data presently available in literature or by initiating large prospective trials. Until this is achieved, the 50\% limit can be used for samples obtained from de novo AML cases, as at this time-point the majority of cells are malignant, where a mutant/total rate above 50\% means the presence of cells with homozygous ITD (47).

The application of home-made molecular genetic tests in the detection and quantitation of FLT3-ITD is rather widespread in scientific literature. On the other hand - to the best of our knowledge - no complex evaluation of analytical properties of the applied methods is published before. In our work 
we defined the precision, inaccuracy, analytical and functional sensitivity, resolution, and ability to detect multiplex ITDs of a PCR+CE-based quantitative method and compared these parameters - where applicable - to that of PCR+AGE/PAGEbased assays. The novel findings concerning the analytical performance of these tests were the following; (i) though the CE-based assay has a very high analytical sensitivity for detection of FLT3-ITD mutant alleles $(0.28 \%)$ the quantitation of the rate of the mutant allele can be done reliably only if it is higher than $1.5 \%$; (ii) the quantitation of the ITD size by $\mathrm{CE}$ can be done with high reproducibility but the accuracy is less perfect (7\% lower ITD-size values compared to DNA sequencing); (iii) the PCR+AGE/PAGE-based methods are not reliable for identification of the presence of multiplex ITDs because of heteroduplex formation. Our data further supports the importance of the proper analytical evaluation of home-made molecular biological diagnostic tests.

In conclusion, our experimental results and data of former publications clearly indicate that a CE-based test seems to be the most suitable method for the detection and quantitation of the mutant/total allele rate of FLT3-ITD, and can be used even for routine diagnostic purposes. This assay can supplement the diagnostics and follow-up of patients with AML. The detection of multiple ITDs and quantitation of the size of the duplication/insertion cannot be suggested for clinical usage at the moment. For research purposes, proper estimation of multiple ITD can be done by CE if the rate of the mutant allele is above $0.28 \%$, and DNA sequencing can provide reliable information about the size and location of ITD.

\section{Acknowledgments}

This work has been supported by the TÁMOP 4.2.1./B-09/1/KONV2010-0007 project that is implemented through the New Hungary Development Plan, co-financed by the European Social Fund. The authors are grateful to Dr. Harjit Pal Bhattoa for the grammatical correction of the manuscript.

\section{Conflict of interest statement}

Authors' conflict of interest disclosure: The authors stated that there are no conflicts of interest regarding the publication of this article. Research funding played no role in the study design; in the collection, analysis and interpretation of data; in the writing of the report; or in the decision to submit the report for publication.

Research funding: None declared.

Employment or leadership: None declared.

Honorarium: None declared.

\section{References}

1. Kappelmayer J, Udvardy M, Antal-Szalmás P. Pgp and FLT3: identification and modulation of two proteins that lead to chemotherapy resistance in acute myeloid leukemia. Curr Med Chem 2007;14:519-30.

2. Schlenk RF, Döhner K. Impact of new prognostic markers in treatment decisions in acute myeloid leukemia. Curr Opin Hematol 2009;16:98-104.
3. Levis M, Small D. FLT3: ITDoes matter in leukemia. Leukemia 2003;17:1738-52.

4. Meshinchi S, Appelbaum FR. Structural and functional alterations of FLT3 in acute myeloid leukemia. Clin Cancer Res 2009;15:4263-9.

5. Yanada M, Matsuo K, Suzuki T, Kiyoi H, Naoe T. Prognostic significance of FLT3 internal tandem duplication and tyrosine kinase domain mutations for acute myeloid leukemia: a metaanalysis. Leukemia 2005;19:1345-9.

6. Beitinjaneh A, Jang S, Roukoz H, Majhail NS. Prognostic significance of FLT3 internal tandem duplication and tyrosine kinase domain mutations in acute promyelocytic leukemia: a systematic review. Leuk Res 2010;34:831-6.

7. Ahmad F, Mandava S, Das BR. Analysis of FLT3-ITD and FLT3Asp835 mutations in de novo acute myeloid leukemia: evaluation of incidence, distribution pattern, correlation with cytogenetics and characterization of internal tandem duplication from Indian population. Cancer Invest 2010;28:63-73.

8. Kiyoi H, Naoe T, Nakano Y, Yokota S, Minami S, Miyawaki S, et al. Prognostic implication of FLT3 and N-RAS gene mutations in acute myeloid leukemia. Blood 1999;93:3074-80.

9. Stirewalt DL, Meshinchi S, Kussick SJ, Sheets KM, PogosovaAgadjanyan E, Willman CL, et al. Novel FLT3 point mutations within exon 14 found in patients with acute myeloid leukaemia. Br J Haematol 2004;124:481-4.

10. Gari M, Abuzenadah A, Chaudhary A, Al-Qahtani M, Banni H, Ahmad W, et al. Detection of FLT3 oncogene mutations in acute myeloid leukemia using conformation sensitive gel electrophoresis. Int J Mol Sci 2008;9:2194-204.

11. Vaughn CP, Elenitoba-Johnson KS. High-resolution melting analysis for detection of internal tandem duplications. J Mol Diagn 2004;6:211-6.

12. DeAngelo DJ, Stone RM, Heaney ML, Nimer SD, Paquette RL, Klisovic RB, et al. Phase 1 clinical results with tandutinib (MLN518), a novel FLT3 antagonist, in patients with acute myelogenous leukemia or high-risk myelodysplastic syndrome: safety, pharmacokinetics, and pharmacodynamics. Blood 2006;108:3674-81.

13. Kayser S, Schlenk RF, Londono MC, Breitenbuecher F, Wittke $\mathrm{K}$, Du J, et al. Insertion of FLT3 internal tandem duplication in the tyrosine kinase domain-1 is associated with resistance to chemotherapy and inferior outcome. Blood 2009;114:2386-92.

14. Schnittger S, Schoch C, Dugas M, Kern W, Staib P, Wuchter C, et al. Analysis of FLT3 length mutations in 1003 patients with acute myeloid leukemia: correlation to cytogenetics, FAB subtype, and prognosis in the AMLCG study and usefulness as a marker for the detection of minimal residual disease. Blood 2002;100:59-66.

15. Fröhling S, Schlenk RF, Breitruck J, Benner A, Kreitmeier $\mathrm{S}$, Tobis K, et al. Prognostic significance of activating FLT3 mutations in younger adults (16-60 years) with acute myeloid leukemia and normal cytogenetics: a study of the AML Study Group Ulm. Blood 2002;100:4372-80.

16. Whitman SP, Archer KJ, Feng L, Baldus C, Becknell B, Carlson $\mathrm{BD}$, et al. Absence of the wild-type allele predicts poor prognosis in adult de novo acute myeloid leukemia with normal cytogenetics and the internal tandem duplication of FLT3: a cancer and leukemia group B study. Cancer Res 2001;61:7233-9.

17. Shih LY, Huang CF, Wu JH, Lin TL, Dunn P, Wang PN, et al. Internal tandem duplication of FLT3 in relapsed acute myeloid leukemia: a comparative analysis of bone marrow samples from 108 adult patients at diagnosis and relapse. Blood 2002;100:2387-92. 
18. Lin MT, Rich RG, Shipley RF, Hafez MJ, Tseng LH, Murphy $\mathrm{KM}$, et al. A molecular fraction collecting tool for the ABI 310 automated sequencer. J Mol Diagn 2007;9:598-603.

19. Shih LY, Kuo MC, Liang DC, Huang CF, Lin TL, Wu JH, et al. Internal tandem duplication and Asp835 mutations of the FMSlike tyrosine kinase 3 (FLT3) gene in acute promyelocytic leukemia. Cancer 2003;98:1206-16.

20. Kottaridis PD, Gale RE, Frew ME, Harrison G, Langabeer SE, Belton AA, et al. The presence of a FLT3 internal tandem duplication in patients with acute myeloid leukemia (AML) adds important prognostic information to cytogenetic risk group and response to the first cycle of chemotherapy: analysis of 854 patients from the United Kingdom Medical Research Council AML 10 and 12 trials. Blood 2001;98:1752-9.

21. Meshinchi S, Stirewalt DL, Alonzo TA, Boggon TJ, Gerbing RB, Rocnik JL, et al. Structural and numerical variation of FLT3/ITD in pediatric AML. Blood 2008;111:4930-3.

22. Gale RE, Green C, Allen C, Mead AJ, Burnett AK, Hills RK, et al. The impact of FLT3 internal tandem duplication mutant level, number, size, and interaction with NPM1 mutations in a large cohort of young adult patients with acute myeloid leukemia. Blood 2008;111:2776-84.

23. Stirewalt DL, Kopecky KJ, Meshinchi S, Engel JH, PogosovaAgadjanyan EL, Linsley J, et al. Size of FLT3 internal tandem duplication has prognostic significance in patients with acute myeloid leukemia. Blood 2006;107:3724-6.

24. Chillón MC, Santamaría C, García-Sanz R, Balanzategui A, María Eugenia S, Alcoceba M, et al. Long FLT3 internal tandem duplications and reduced PML-RARalpha expression at diagnosis characterize a high-risk subgroup of acute promyelocytic leukemia patients. Haematologica 2010;95:745-51.

25. Zwaan CM, Meshinchi S, Radich JP, Veerman AJ, Huismans DR, Munske L, et al. FLT3 internal tandem duplication in 234 children with acute myeloid leukemia: prognostic significance and relation to cellular drug resistance. Blood 2003;102: 2387-94.

26. Meshinchi S, Alonzo TA, Stirewalt DL, Zwaan M, Zimmerman M, Reinhardt D, et al. Clinical implications of FLT3 mutations in pediatric AML. Blood 2006;108:3654-61.

27. Thiede C, Steudel C, Mohr B, Schaich M, Schäkel U, Platzbecker U, et al. Analysis of FLT3-activating mutations in 979 patients with acute myelogenous leukemia: association with FAB subtypes and identification of subgroups with poor prognosis. Blood 2002;99:4326-35.

28. Whitman SP, Maharry K, Radmacher MD, Becker H, Mrózek $\mathrm{K}$, Margeson D, et al. FLT3 internal tandem duplication associates with adverse outcome and gene- and microRNA-expression signatures in patients 60 years of age or older with primary cytogenetically normal acute myeloid leukemia: a Cancer and Leukemia Group B study. Blood 2010;116:3622-6.

29. Liang DC, Shih LY, Hung IJ, Yang CP, Chen SH, Jaing TH, et al. Clinical relevance of internal tandem duplication of the FLT3 gene in childhood acute myeloid leukemia. Cancer 2002;94:3292-8.

30. Mills KI, Gilkes AF, Walsh V, Sweeney M, Gale R. Rapid and sensitive detection of internal tandem duplication and activating loop mutations of FLT3. Br J Haematol 2005;130:203-8.

31. Scholl S, Salzmann S, Kaufmann AM, Höffken K. Flt3-ITD mutations can generate leukaemia specific neoepitopes: potential role for immunotherapeutic approaches. Leuk Lymphoma 2006;47:307-12.

32. Brown P, Meshinchi S, Levis M, Alonzo TA, Gerbing R, Lange B, et al. Pediatric AML primary samples with FLT3/ITD mutations are preferentially killed by FLT3 inhibition. Blood 2004;104:1841-9.

33. Seedhouse CH, Pallis M, Grundy M, Shang S, Russell NH. FLT3-ITD expression levels and their effect on STAT5 in AML with and without NPM mutations. $\mathrm{Br} \mathrm{J}$ Haematol 2009;147:653-61.

34. Stirewalt DL, Willman CL, Radich JP. Quantitative, real-time polymerase chain reactions for FLT3 internal tandem duplications are highly sensitive and specific. Leuk Res 2001;25:1085-8.

35. Chou WC, Hou HA, Liu CY, Chen CY, Lin LI, Huang YN, et al. Sensitive measurement of quantity dynamics of FLT3 internal tandem duplication at early time points provides prognostic information. Ann Oncol 2011;22:696-704.

36. Yamamoto Y, Kiyoi H, Nakano Y, Suzuki R, Kodera Y, Miyawaki S, et al. Activating mutation of D835 within the activation loop of FLT3 in human hematologic malignancies. Blood 2001;97:2434-9.

37. Lin P, Jones D, Medeiros LJ, Chen W, Vega-Vazquez F, Luthra R. Activating FLT3 mutations are detectable in chronic and blast phase of chronic myeloproliferative disorders other than chronic myeloid leukemia. Am J Clin Pathol 2006;126:530-3.

38. Shih LY, Huang CF, Wang PN, Wu JH, Lin TL, Dunn P, et al. Acquisition of FLT3 or N-ras mutations is frequently associated with progression of myelodysplastic syndrome to acute myeloid leukemia. Leukemia 2004;18:466-75.

39. Ravandi F, Kantarjian H, Faderl S, Garcia-Manero G, O'Brien $\mathrm{S}$, Koller C, et al. Outcome of patients with FLT3-mutated acute myeloid leukemia in first relapse. Leuk Res 2010;34:752-6.

40. Cloos J, Goemans BF, Hess CJ, van Oostveen JW, Waisfisz Q, Corthals S, et al. Stability and prognostic influence of FLT3 mutations in paired initial and relapsed AML samples. Leukemia 2006;20:1217-20.

41. Huang Q, Chen W, Gaal KK, Slovak ML, Stein A, Weiss LM. A rapid, one step assay for simultaneous detection of FLT3/ITD and NPM1 mutations in AML with normal cytogenetics. Br J Haematol 2008;142:489-92.

42. Murphy KM, Levis M, Hafez MJ, Geiger T, Cooper LC, Smith $\mathrm{BD}$, et al. Detection of FLT3 internal tandem duplication and D835 mutations by a multiplex polymerase chain reaction and capillary electrophoresis assay. J Mol Diagn 2003;5:96-102.

43. Libura M, Asnafi V, Tu A, Delabesse E, Tigaud I, Cymbalista F, et al. FLT3 and MLL intragenic abnormalities in AML reflect a common category of genotoxic stress. Blood 2003;102:2198-204.

44. Ciolli S, Vannucchi AM, Leoni F, Nozzoli C, Longo G, Salati A, et al. Internal tandem duplications of FLT3 gene (FLT3/ITD) predicts a poor post-remission outcome in adult patients with acute non-promyelocytic leukemia. Leuk Lymphoma 2004;45:73-8.

45. Noguera NI, Ammatuna E, Zangrilli D, Lavorgna S, Divona M, Buccisano F, et al. Simultaneous detection of NPM1 and FLT3ITD mutations by capillary electrophoresis in acute myeloid leukemia. Leukemia 2005;19:1479-82.

46. Knapper S, Mills KI, Gilkes AF, Austin SJ, Walsh V, Burnett AK. The effects of lestaurtinib (CEP701) and PKC412 on primary AML blasts: the induction of cytotoxicity varies with dependence on FLT3 signaling in both FLT3-mutated and wild-type cases. Blood 2006;108:3494-503.

47. Green C, Linch DC, Gale RE. Most acute myeloid leukaemia patients with intermediate mutant FLT3/ITD levels do not have detectable bi-allelic disease, indicating that heterozygous disease alone is associated with an adverse outcome. Br J Haematol 2008;142:423-6. 